\title{
Highly efficient fluorescence detection in picoliter volume liquid- core waveguides
}

Aaron R. Hawkins

hawkins@ee.byu.edu

John P. Barber

johnpbarber@yahoo.com

Dongliang Yin

Follow this and additional works at: https://scholarsarchive.byu.edu/facpub

Part of the Electrical and Computer Engineering Commons

\section{Original Publication Citation}

Yin, Dongliang, John P. Barber, Aaron R. Hawkins, and Holger Schmidt. "Highly efficient fluorescence detection in picoliter volume liquid-core waveguides." Applied Physics Letters 87 (25)

\section{BYU ScholarsArchive Citation}

Hawkins, Aaron R.; Barber, John P.; and Yin, Dongliang, "Highly efficient fluorescence detection in picoliter volume liquid-core waveguides" (2005). Faculty Publications. 340.

https://scholarsarchive.byu.edu/facpub/340

This Peer-Reviewed Article is brought to you for free and open access by BYU ScholarsArchive. It has been accepted for inclusion in Faculty Publications by an authorized administrator of BYU ScholarsArchive. For more information, please contact ellen_amatangelo@byu.edu. 


\title{
Highly efficient fluorescence detection in picoliter volume liquid-core waveguides
}

\author{
Dongliang Yin ${ }^{\text {a) }}$ \\ School of Engineering, University of California, Santa Cruz, 1156 High Street, Santa Cruz, \\ California 95064 \\ John P. Barber and Aaron R. Hawkins \\ Department of Electrical and Computer Engineering, Brigham Young University, 459 Clyde Building, \\ Provo, Utah 84604
}

Holger Schmidt

School of Engineering, University of California, Santa Cruz, 1156 High Street, Santa Cruz, California 95064

(Received 24 January 2005; accepted 13 October 2005; published online 17 November 2005)

\begin{abstract}
We report loss improvement and fluorescence detection in integrated antiresonant reflecting optical waveguides with liquid cores. The minimum waveguide loss is reduced to $0.33 / \mathrm{cm}$ by compensating for thickness variations in the fabrication process. We demonstrate fluorescence detection from as few as 490 molecules in a $57 \mathrm{pl}$ core using these optimized waveguides. We measure angular fluorescence collection factors as high as $15 \%$ per facet in good agreement with theory. This demonstrates the potential of integrated hollow-core waveguides as optical sensors for single-molecule spectroscopy. (c) 2005 American Institute of Physics. [DOI: 10.1063/1.2135378]
\end{abstract}

Optical fluorescence is one of the most commonly used analytical tools in biology and medicine. Several bulk fluorescence techniques with single molecule sensitivity exist, including confocal microscopy, epifluorescence, near-field scanning microscopy, and evanescent field detection. ${ }^{1}$ A fully integrated approach to single molecule spectroscopy is desirable due to the inherent advantages of integrated optics such as compact size, robustness, inexpensive fabrication, and integration with other functional elements such as wavelength filtering ${ }^{2}$ and microfluidic systems. A fluorescence sensor with fully planar beam paths for single molecules in solution requires the ability to guide light through the solvent (typically water, index $\sim 1.33$ ) and to excite very small sample volumes on the order of picoliters or below to reduce background fluorescence levels. ${ }^{3}$ Index guiding is possible using Teflon AF waveguides with cladding indexes as low as 1.29. However, such waveguides have large cross sections that preclude achieving the required small excitation volumes. ${ }^{5}$ Multilayer structures based on photonic crystals ${ }^{6,7}$ or antiresonant reflecting optical waveguides ${ }^{8}$ (ARROWs) are alternatives that can be used for building micron-sized channels that guide both light and liquid sample material. ${ }^{9}$ Fluorescence from multimode ARROW waveguides with large cross sections $(150 \times 150 \mu \mathrm{m}$, volume $0.2 \mu \mathrm{l}$ for $1 \mathrm{~cm}$ length $)$ fabricated with a wafer-bonding process has been reported. ${ }^{10} \mathrm{We}$ have recently demonstrated light confinement and low-loss propagation in liquid and hollow-core ARROWs with picoliter volumes that can be fabricated using standard silicon technology ${ }^{11,12}$ without the need for wafer bonding. The waveguides can be arranged in two-dimensional planar waveguide arrays on a semiconductor chip. ${ }^{2}$ Due to their small mode cross section on the order of a few $\mu \mathrm{m}^{2}$, they are ideal candidates for single molecule spectroscopy. In this letter, first we report significant reduction of the waveguide loss by a modified fabrication process. Second we present the

\footnotetext{
${ }^{a)}$ Electronic mail: yindl@soe.ucsc.edu
}

first demonstration of efficient fluorescence detection from dye molecules in liquid-core ARROWs with cross-section dimensions below $10 \mu \mathrm{m}$ and picoliter volumes. We determine the fluorescence collection efficiency experimentally and theoretically, and show that the radiation is captured efficiently in these waveguides.

ARROW waveguides are based on fulfilling the antiresonant reflection condition for a leaky waveguide mode in each cladding layer. ${ }^{8}$ Figure 1(a) shows the cross section of a liquid-core ARROW where a liquid core with typical dimensions of $3.5 \times 10 \mu \mathrm{m}$ is surrounded by three periods of alternating $\mathrm{SiN} / \mathrm{SiO}_{2}$ layers. ${ }^{12}$ The total waveguide loss for $x$-polarized light can be separated to a very good approximation into the sum of the one-dimensional losses for a $p$-wave in the transverse and an $s$-wave in the lateral directions, respectively, as shown in Fig. 1(b). ${ }^{2}$ The loss is dominated by the $p$-wave contribution in the direction of the incident polarization. Loss in our geometry can, therefore, be minimized by optimizing the cladding layer structure in the lateral $(x)$ direction.

Due to the plasma-enhanced chemical-vapor deposition (PECVD) deposition process, the thickness of the vertical
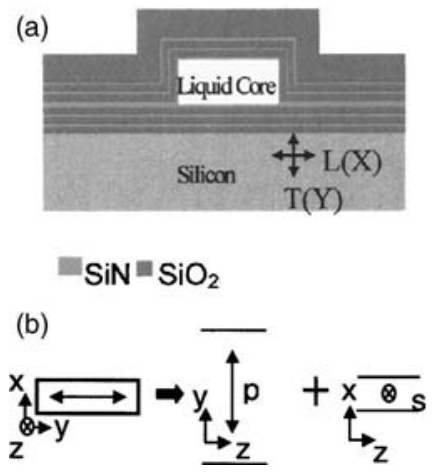

FIG. 1. (a) Cross section of the ARROW waveguide. L: lateral; T: transverse. (b) Schematic separation of mode loss. 


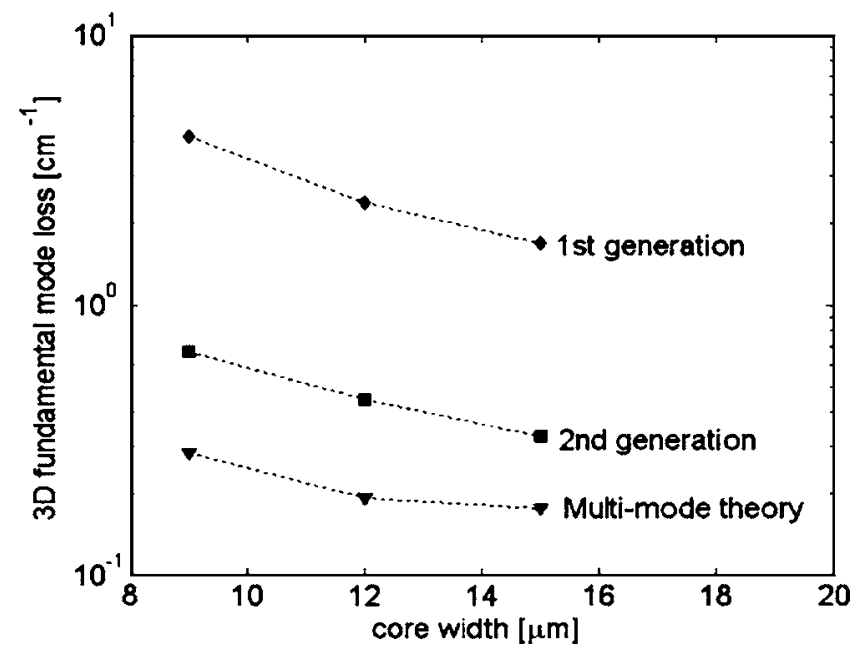

FIG. 2. Waveguide loss vs ARROW core width. Diamonds and squares: experimental results; triangles: calculation.

ARROW layers for lateral confinement is approximately 1.25 times thinner than that of the horizontal layers for transverse confinement. This is one of the main sources of the difference in waveguide loss between theory and experiment of our first generation ARROWs. ${ }^{12}$ To compensate for the reduced thickness in the lateral direction, we grew the upper ARROW layers 1.25 times thicker to minimize loss in the lateral direction with little effect on loss in the transverse direction. Figure 2 shows the waveguide loss at $633 \mathrm{~nm}$ measured with the same technique as in Ref. 12. The input polarization for all experiments presented here is along the $x$ direction. We find a significant reduction by a factor of 5 to 6 in waveguide loss between first- and second-generation liquid-core ARROWs. The minimum waveguide loss of the second generation is $0.33 / \mathrm{cm}$ for a $15-\mu \mathrm{m}$-wide core compared to $1.7 / \mathrm{cm}$ for the first generation with the same cross section, ${ }^{12}$ and agrees more closely with theoretical calculations (triangles) that include higher-order lateral modes. The remaining discrepancy is likely due to scattering and surface roughness of the ARROW layers.

We used these optimized waveguides to demonstrate the use of liquid-core ARROWs for highly sensitive fluorescence detection. Figure 3(a) shows the schematic of the experimental setup. The pump beam from a laser diode (LD) passes through an excitation filter (F0, bandpass at $633 \mathrm{~nm}$ ), and is then coupled into a single mode fiber aligned with the center of the ARROW core. The core is filled with fluorescent Alexa 647 molecules in ethylene glycol solution. A lock-in amplifier is used to reduce the background noise. Two fluorescence filters (F1, F2; bandpass at 650-700 nm) are placed between the output of the waveguide and a photomultiplier tube (PMT) detector. A beam splitter (BS) is used to split the output between the PMT and an optical multimeter (OM), respectively. The PMT collects the fluorescence only and the OM detects essentially the output at the pump wavelength (the pump power is significantly higher than the fluorescence power). The concentration-dependent fluorescence output power is shown in Fig. 3(b) for a waveguide with $3.5 \mu \mathrm{m}$ $\times 9 \mu \mathrm{m}$ core, and $1.8 \mathrm{~mm}$ length (sample volume $57 \mathrm{pl}$ ), and the resulting fluorescence spectrum taken with an optical spectrum analyzer. The pump power was kept at a few microwatts to minimize photobleaching. The detected power decreases linearly with the concentration in agreement with Downloaded 13 Feb 2009 to 128.187.0.164. Redistribution subject (a)
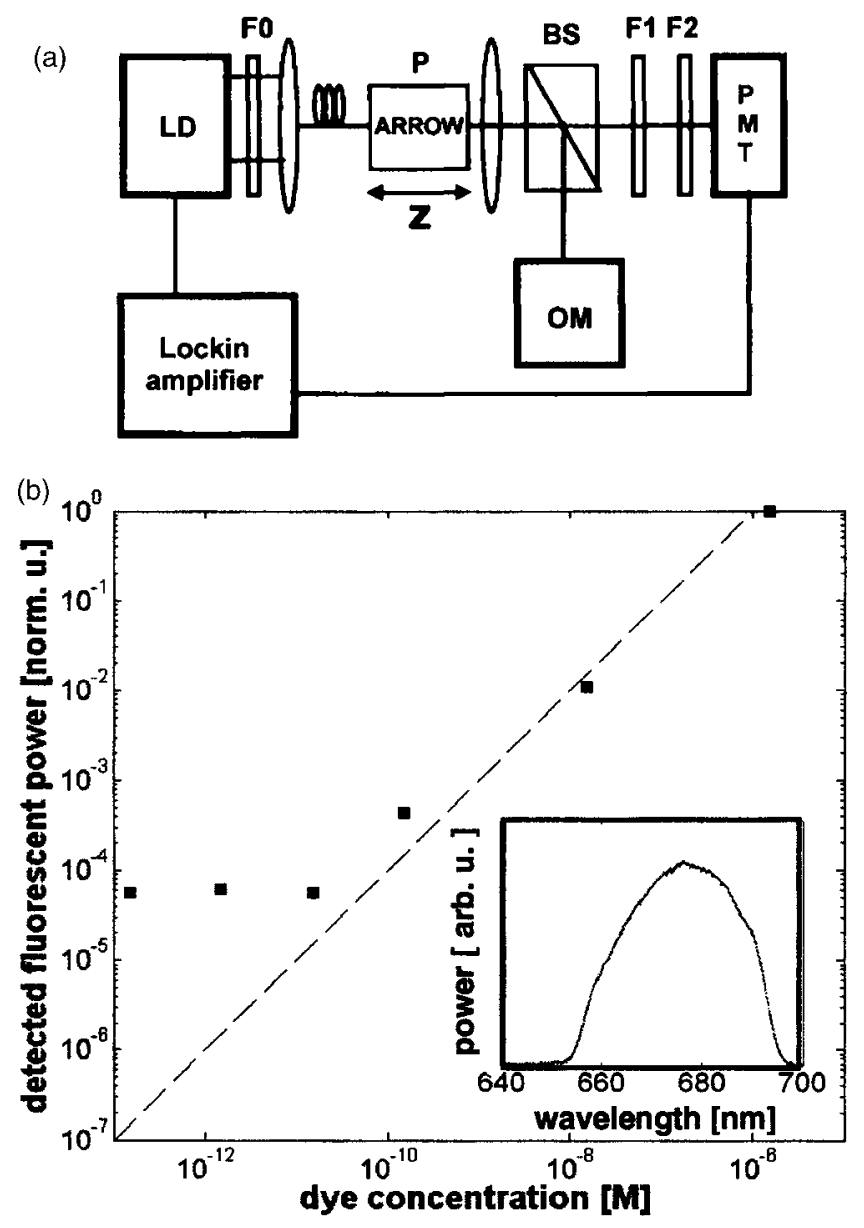

FIG. 3. (a) Schematic graph of the fluorescence detection setup: LD: Laser diode, $P$ : polarization controller; BS: beam splitter; F0, F1, F2: filters. (b) Normalized fluorescence power vs dye concentration; circles: experiment; dashed line: theory. Inset: fluorescence spectrum taken at $c=10^{-4} \mathrm{M}$.

the Lambert-Beer law ${ }^{1}$ until the detection limit is reached. We were able to detect concentrations as low as $10^{-11} \mathrm{M}$, corresponding to 490 molecules in the core. The detectable concentration in this collinear setup is limited by the detector sensitivity, solvent contributions ${ }^{1}$ and by residual power at the pump wavelength that leaks through the fluorescence filters F1 and F2. Single molecule sensitivity can be achieved by using an avalanche photodiode (APD) detector and perpendicular beam paths for the excitation and fluorescence signals on the chip. ${ }^{2}$

Next, we determined the angular collection factor $F_{\text {coll }}$ of the ARROW waveguide. In confocal microscopy, $F_{\text {coll }}$ is determined by the numerical aperture of the objective. In our case, it is given by the ratio of fluorescence power emitted by the dye molecules $\left(P_{f}\right)$ and detectable fluorescence power at the waveguide output $\left(P_{\mathrm{df}}\right)$,

$$
F_{\mathrm{coll}}=\frac{P_{\mathrm{df}}}{P_{f}}=\frac{P_{\mathrm{df}} \alpha_{P}}{\frac{\lambda_{P}}{2 \lambda_{f}} \eta P_{P}(L) \sigma N\left(e^{\alpha_{P} L}-1\right)} .
$$

Here, $\alpha_{p}$ is the waveguide loss at the pump wavelength; $\eta$, $N$, and $\sigma$ are the fluorescence quantum efficiency, density, and absorption cross section ${ }^{13}$ of the dye molecules, respectively; and $P_{P}(L)$ is the detected pump power. The last expression in Eq. (1) relates the generated fluorescence power to the detected pump power and takes into account pump
to AIP license or copyright; see http://apl.aip.org/apl/copyright.jsp 


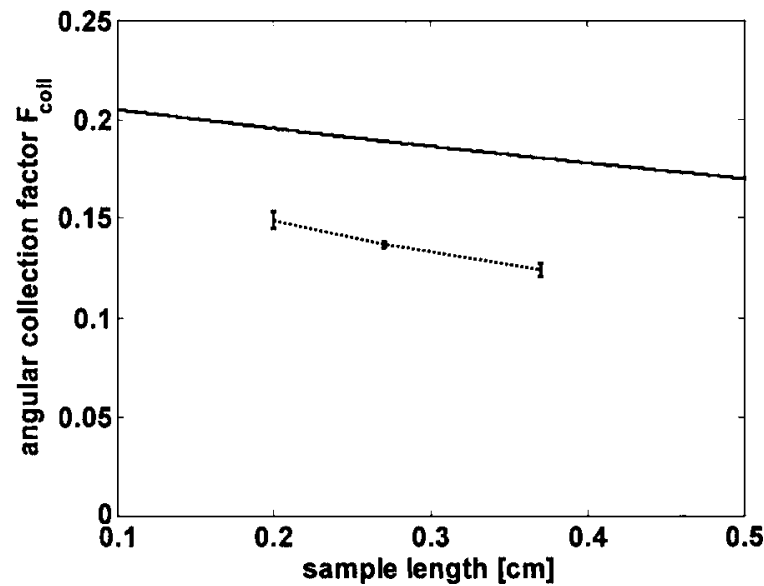

FIG. 4. Fluorescence collection factor vs ARROW waveguide length; dotted line: experiment, solid line: theory. Error bars are obtained from repeated measurements of $F_{\text {coll }}$.

attenuation due to waveguide loss $\alpha_{P}$, and the fact that the fluorescence propagates in both the positive and negative $z$ directions and the pump beam only in a forward direction. It allows us to calculate $F_{\text {coll }}$ from the experimentally observed values after correcting for the signal attenuation due to the fluorescence filters F1 and F2, and the asymmetric splitting ratio of the beamsplitter $(1: 0.887)$. We measured the collection factor as a function of sample length for a waveguide with $w=9 \mu \mathrm{m}$ and a dye concentration of $1.5 \times 10^{-8} \mathrm{M}$ (dotted symbols in Fig. 4). $F_{\text {coll }}$ decreases slowly with sample length due to the presence of waveguide loss, but collection is very efficient with values as high as $15 \%$ per facet.

We carried out numerical simulations of the collection efficiency to verify the experimental value. To this end, we assume a homogeneous distribution of dye molecules with randomly oriented oscillating dipoles throughout the core. For a molecule at a longitudinal position $z, P_{\mathrm{df}}$ is determined by the fraction of power that is initially coupled into propagating low-loss ARROW modes, and the fraction of power in these modes that appears at the waveguide output in the presence of the waveguide loss. Using a commercial software package (OMNISIM, OPhoton Design), we first calculate the polarization-dependent coupling of fluorescence into the lower-order ARROW modes (typically the first three odd modes). Higher-order leaky ARROW modes with higher loss quickly escape from the core (within $<100 \mu \mathrm{m}$ ) and can be neglected for waveguides longer than $1 \mathrm{~mm}$. We then determine the intensity remaining after propagating to the output of the waveguide in presence of the experimentally determined waveguide loss. Because the fluorescence is only partially polarized due to random orientation and rotation of the dye molecules, $X, Y$, and $Z$ polarization components [as defined in Fig. 1(b) and Fig. 2, measured ratio $X: Y: Z$ $=1.9: 1: 1]$ are combined to deduce the collection factor. ${ }^{14}$ The remaining intensity is integrated over the length $\mathbf{L}$ of the waveguide to account for all possible longitudinal molecule positions. The calculated $F_{\text {coll }}$ are shown as the solid line in Fig. 4 and show good agreement with the experimental data. The discrepancy arises from the finite number of modes taken into account in the numerical simulation, and the fact that fluorescence quenching ${ }^{15}$ and photobleaching were neglected. Finally, we assumed a quantum yield of $\eta=1$. Collection factors of other fluorescence detection schemes with high numerical aperture can be significantly larger. However, the total collection efficiency $D$ of a system also includes other factors such as transmission losses, filter response, and detector quantum efficiency, resulting in typical values for $D$ of $1-8 \%{ }^{1,16}$ One of the advantages of the integrated ARROW approach is that some factors that reduce $F_{\text {coll }}$ to $D$ can be eliminated. For example, wavelength filtering can be integrated on the chip $^{2}$ and the signal can be fed very efficiently to an APD detector via coupling into multimode fiber right at the ARROW output. Consequently, total collection efficiencies comparable with typical microscopy setups can be expected.

In summary, we have presented loss improvement and fluorescence detection in integrated liquid-core waveguides with picoliter volumes. Fluorescence collection factors in excess of $15 \%$ per waveguide facet were measured and modeled. The sensitivity can be further improved by reducing the excitation volume using perpendicular excitation and collection paths, and by optimizing the cladding layers for high loss at the pump wavelength. ${ }^{2}$ This will allow the use of liquid-core ARROW waveguides as fully integrated optical fluorescence or Raman sensors with single molecule sensitivity.

The authors acknowledge stimulating discussions with R. Ram, T. Huser, and D. Deamer, and support from the National Institutes of Health under Award No. R21EB003420-01. H.S. acknowledges support through NSF Career Award No. ECS-0131945.

${ }^{1}$ W. E. Moerner and David P. Fromm, Rev. Sci. Instrum. 74, 3597 (2003). ${ }^{2}$ H. Schmidt, D. Yin, J. P. Barber, and A. R. Hawkins, IEEE J. Sel. Top. Quantum Electron. 11, 519 (2005).

${ }^{3}$ C. Zander, J. Enderlein, and R. Keller, Single-Molecule Detection in Solution (Wiley, New York, 2002).

${ }^{4}$ J. H. Lowry, J. S. Mendelowitz, and N. S. Subramanian, Opt. Eng. (Bellingham) 31, 1982 (1992).

${ }^{5}$ A. Datta, I. Eom, A. Dhar, P. Kuban, R. Manor, I. Ahmad, S. Gangopadhyay, T. Dallas, M. Holtz, H. Temkin, and P. Dasgupta, IEEE Sens. J. 3, 788 (2003).

${ }^{6}$ Y. Fink, J. N. Winn, S. Fan, C. Chen, J. Michel, J. D. Joannopoulos, and E. L. Thomas, Science 282, 1679 (1998).

${ }^{7}$ P. Russell, Laser Focus World 38, 77 (2002).

${ }^{8}$ M. A. Duguay, Y. Kokubun, T. Koch, and L. Pfeiffer, Appl. Phys. Lett. 49, 13 (1986).

${ }^{9}$ T. Delonges and H. Fouckhardt, J. Chromatogr., A 716, 135 (1995).

${ }^{10}$ S. Campopiano, R. Bernini, L. Zeni, and P. M. Sarro, Opt. Lett. 29, 1894 (2004).

${ }^{11}$ D. Yin, J. P. Barber, A. R. Hawkins, and H. Schmidt, Opt. Express 12, 2710 (2004).

${ }^{12}$ D. Yin, D. W. Deamer, H. Schmidt, J. P. Barber, and A. R. Hawkins, Appl. Phys. Lett. 85, 3477 (2004).

${ }^{13} \mathrm{http}: / /$ www.probes.com

${ }^{14}$ A. J. Pesce, C. Rosen, and T. L. Pasby, Fluorescence Spectroscopy (Dekker, New York, 1971).

${ }^{15}$ Jan Slavik, Fluorescent Probes in Cellular and Molecular Biology (CRC Press, Boca Raton, FL, 1994).

${ }^{16}$ J. R. Fries, L. Brand, C. Eggeling, M. Kollner, and C. A. M. Seidel, J. Phys. Chem. 102, 6601 (1998). 